\title{
Effects of intensive short-term dynamic psychotherapy on social cognition in major depression
}

\author{
Bita Ajilchi ${ }^{1}$, Steve Kisely ${ }^{2,3}$, Vahid Nejati ${ }^{4}$ and Jon Frederickson ${ }^{5}$ \\ ${ }^{1}$ Department of Psychology, Faculty of Human Science, Science and Research Branch, Islamic Azad University (IAU), Tehran, Iran, ${ }^{2}$ Community \\ Health and Epidemiology, Dalhousie University, Halifax, NS, Canada, ${ }^{3}$ Rural and Southern Clinical Schools, University of Queensland, \\ Woolloongabba, Australia, ${ }^{4}$ Department of Psychology, Faculty of Human Science, Shahid Beheshti University, Tehran, Iran, and ${ }^{5}$ MSW Faculty, \\ Washington School of Psychiatry, Washington, DC, USA
}

\begin{abstract}
Background: Social cognition is commonly affected in psychiatric disorders and is a determinant of quality of life. However, there are few studies of treatment.

Objective: To investigate the efficacy of intensive short-term dynamic psychotherapy on social cognition in major depression.

Method: This study used a parallel group randomized control design to compare pre-test and post-test social cognition scores between depressed participants receiving ISTDP and those allocated to a wait-list control group. Participants were adults (19-40 years of age) who were diagnosed with depression. We recruited 32 individuals, with 16 participants allocated to the ISTDP and control groups, respectively. Both groups were similar in terms of age, sex and educational level.

Results: Multivariate analysis of variance (MANOVA) demonstrated that the intervention was effective in terms of the total score of social cognition: the experimental group had a significant increase in the post-test compared to the control group. In addition, the experimental group showed a significant reduction in the negative subjective score compared to the control group as well as an improvement in response to positive neutral and negative states.

Conclusion: Depressed patients receiving ISTDP show a significant improvement in social cognition post treatment compared to a wait-list control group.
\end{abstract}

\section{Keywords}

Depression, social cognition, intensive short-term dynamic psychotherapy (ISTDP)

\section{History}

Received 5 June 2017

Revised 17 November 2017

Accepted 26 February 2018

Published online 23 May 2018

\section{Introduction}

Social cognition focuses on the identification and interpretation of other people's affect, mental states and actions. This requires the knowledge of social cues and rules to manage emotional responses and maintain interpersonal relationships (Pinkham et al., 2014). These functions are often impaired in psychiatric disorders, especially in schizophrenia (Weightman et al., 2014), autism (Weightman et al., 2014) and bipolar affective disorder (Derntl et al., 2009; Gray et al., 2006), but less so in depression (Wang et al., 2008; Weightman et al., 2014). Even so, patients with depression show deficits in emotion recognition with a bias toward recognizing negative states of at least a moderate overall effect size (Weightman et al., 2014). For example, they tend not to recognize happy faces and interpret neutral ones as sad (Billeke et al., 2013). The mechanism appears to be an increased sensitivity toward sad expressions in comparison with happy ones rather than diminished accuracy of recognition (Weightman et al., 2014).

Correspondence: Bita Ajilchi, Department of Psychology, Faculty of Human Science, Sciences and Research Branch, Islamic Azad University (IAU), Tehran, Iran. E-mail: ajilchi_b@yahoo.com
Given the effects on patients' quality of life (Billeke et al., 2013; Cusi et al., 2012), these deficits can be a focus of treatment outcomes. Treatments usually focus on the emotional domain, particularly "theory of mind" (ToM). The ToM is defined as "the ability to attribute beliefs, desires, intentions and emotions to oneself and to others to explain and predict behavior" (Lahera et al., 2013). One measurement tool of adult social sensitivity is the "reading the mind in the eyes" test (RMET) (Baron-Cohen et al., 2001a). Evidence suggests that this capacity is impaired in depressed patients (Kerr et al., 2003).

For instance, Lee et al. (2005) reported that women with unipolar depression showed deficits in mind-reading tasks and had lower scores on reading of positive images, possibly due to cognitive impairment in processing positive stimuli (Beevers et al., 2009).

In a study by Nejati et al. (2012a), 45 patients with major depression were compared with 50 healthy counterparts using a mind-reading test through eye images. Patients with major depression had a significantly lower ability to perceive positive or neutral mental states compared to their normal counterparts, but, conversely, they had a higher ability to perceive negative mental states. 
Neuroimaging studies of social cognition in patients with mood disorders reveal enhanced activation in limbic and emotion-related structures and attenuated activity within frontal regions associated with emotion regulation and higher cognitive functions (Cusi et al., 2012). These results suggest an overall lack of inhibition by higher-order cognitive structures on limbic and emotion-related structures during social cognitive processing in patients with mood disorders. Preliminary evidence also indicates that psychotropic medications and CBT may exert different effects on the neural networks involved in affect recognition. Specifically, psychotropic medication attenuates levels of activation in the prefrontal and subcortical regions involved in affective processing while CBT increases levels of neural activation in regions associated with emotion regulation and higherorder cognitive processing.

Even though social cognition is a notable component in major depression, and we understand its neurological correlates, the ability to improve social cognition remains quite limited. Only one study investigated the effect of social cognition and interaction training (SCIT) for 18 weeks in outpatients with bipolar and schizoaffective disorder who had impairment in social cognition when unwell (Lahera et al., 2013). Training significantly improved emotion perception and depression symptoms and social cognition in the short, although longer-term effects were unknown. However, this study did not include patients with major depression. To explore another means of improving social cognition, we tested the hypothesis of whether improving affect awareness and experience internally would lead to improved interpersonal social cognition in patients with depression.

We used intensive short-term dynamic psychotherapy (ISTDP) because no previous studies have assessed the effects of this approach on social cognition in depressed individuals. The ISTDP is delivered weekly with an average of less than 40 sessions (Davanloo, 2000; Davanloo, 2005). It has been shown to be effective in a wide range of common mental disorders (Abbass et al., 2012; Town \& Driessen, 2013). Patients are enabled to experience and tolerate painful affects associated with traumatic attachment experiences.

We therefore examined whether ISTDP might produce significant changes in social cognition. This article formed part of a study examining the effects of ISTDP on a range of psychiatric outcomes (Ajilchi et al., 2016). We hypothesized that with increased awareness and experience of feelings resulting from ISTDP psychotherapy, patients would experience improved social cognition.

\section{Methods}

\section{Design}

Using a randomized controlled design, participants meeting DSM-IV criteria for major depressive episode were allocated randomly to either the control group (wait-list) or an experimental group (ISTDP). A clinic secretary who had no other involvement in the design or implementation of the research procedure conducted the randomization procedure. We tried to recruit 20 subjects for each group. Participants' depression severity and social cognition were assessed at two time points, baseline (prior to allocation) and after the experimental/control intervention in order to compare group differences.

\section{Participants}

Consecutive referrals, aged 19-40 years, in 2011 who were referred to a mental health outpatient psychotherapy clinic in Tehran, Iran, were screened for inclusion in the study. Participants were evaluated by a clinical psychologist and were included if they met DSM-IV criteria for current major depressive episode, had a depression severity score of over 20 on the Beck Depression Inventory (BDI)-II, were not currently receiving antidepressant treatment and provided informed written consent. We chose the BDI as it has established reliability and validity in Iranian patients (Kaviani et al., 2001). Participants were excluded if they had bipolar disorder, psychosis, eating disorder, drug abuse or severe suicidality. In total, 40 participants were included and allocated to a group.

\section{Treatment}

The ISTDP begins with an evaluation of psychological capacities such as unconscious anxiety discharge pathways and defensive patterns and the capacity to respond to treatment (Abbass, 2015). Patients can, and often do, defend against painful realities, anxiety-provoking feelings and close emotional contact with others, including the therapist (Coughlin, 2017, p. 75). The patient is then encouraged to re-experience unprocessed feelings about recent and past traumatic events followed by recapitulation of what was learned so as to weaken the avoidance of emotional experiences and interpersonal closeness. Common emphases in depression tend to be working through grief about losses and the experience and processing of buried rage and guilt related to attachment trauma in childhood (Abbass, 2015).

Termination was determined by the therapist and patient based on treatment progress (Ajilchi et al., 2016). The average number of sessions in this trial was 15 . The first was a trial of therapy, lasting 90 minutes, and the remaining sessions were 60 minutes in length. Therapists were two registered psychologists with between 2 and 4 years of supervision in ISTDP at the time of the trial (Ajilchi et al., 2016). Treatment sessions were videotaped, recorded and reviewed regularly with an experienced supervisor to ensure treatment adherence (Ajilchi et al., 2016).

\section{Outcome measures}

Measure of social cognition: the reading mind in eyes test

This neuropsychological test of mind-reading was developed by Baron-Cohen et al. (2001b). Participants are presented with 36 images of pairs of eyes and asked to choose one of four emotional states they think best explains the photo (Figure 1). The test has been translated into Farsi with demonstrated reliability and used in multiple studies in Iran (Nejati et al., 2012a,b). When used in assessing depression, the emotional states can be divided into those that are positive, neutral or negative, and the results analyzed in terms of whether participants are more likely to recognize states from one or more of these three categories. This 


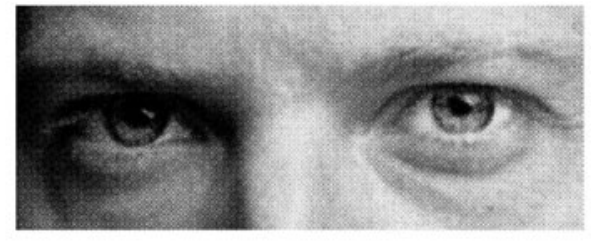

\section{Irritated Disappointed Depressed Accusing}

Figure 1. A sample picture from the Baron-Cohen mind-reading test (Correct answer: Accusing). (Baron-Cohen et al., 2001a).

Table 1. Scoring interpretation of images in Baron-Cohen test (2001a).

\begin{tabular}{lccc}
\hline Image response & Positive & Neutral & Negative \\
\hline Positive & 0 & +1 & +2 \\
Neutral & -1 & 0 & +1 \\
Negative & -2 & -1 & 0 \\
\hline
\end{tabular}

neuropsychological test has been used in many studies for investigating the ability of both healthy and patient populations to infer the mental state of others (Wang et al., 2008).

In this study, the 36 images were divided into those of positive, neutral and negative emotional tone (Nejati et al., 2013). During the test, participants were asked to identify which emotional group best represented a particular image. Agreement between the perceived and objective emotional tone of the photo scored zero (Table 1). However, if a participant scored a photo with a neutral tone as being positive, the score was +1 , and if it was seen as being negative, -1 (Table 1). Similarly, neutral and positive impressions of a negative photo scored +1 and +2 , respectively, while conversely, negative and neutral interpretations of a positive image scored -1 and -2 (Table 1). As a result, negative scores represented worsening depression, and positive scores the brightening of mood.

We used the RMET for the following reasons. First, faces represent not only a complex class of visual patterns, but also have social meaning (e.g. Pelphrey et al., 2004). Second, measures used in neuropsychological evaluation should be based on brain structure and function, and the perception of faces is linked to a well-known anatomical area in the brain (the fusiform gyrus) (Weiner \& Grill-Spector, 2012). Finally, the RMET is less dependent on the previous experiences of individuals than traditional behavioral tasks.

\section{Statistical analysis}

As this was a pilot study, sample size calculations were not performed. We performed analyses on the per-protocol sample because missing data prevented an intention-to-treat (ITT) analysis. We initially examined differences between the groups in terms of demographic and clinical characteristics using $t$ - and chi-square tests. We then used one-way multivariate analysis of covariance (MANCOVA) to account for differences in baseline functioning when examining the effects of treatment on social cognition We calculated the effect size based on partial $\eta^{2}$ values to reflect the proportion of variance attributable to the dependent variable after controlling for other predictors (Ajilchi et al., 2016). A small effect is greater than 0.14 , medium effect greater than 0.36 and a large effect greater than 0.51 (Leech et al., 2005).

\section{Results \\ Participants}

From September 2011 to October 2012, participants were recruited from a mental health outpatient clinic. In total, 40 participants were included and allocated to either the treatment or control group. From this sample, four patients from each group dropped out of the study. Therefore, final measures were not collected from these participants. The reason for dropout in the control group was that patients preferred not to wait for treatment. In the ISTDP group, two patients were referred for alternative treatment after the first session. In one patient, this was because of low psychological mindedness, another preferred antidepressant treatment and the third terminated due to a change in location. It was unclear why the last patient dropped out. The final sample of those who completed the treatment was $32(N=16$ ISTDP; $N=16$ wait list).

Twenty-three out of the 32 participants were female (72\%) with an average age of 22 . Twenty-two (68\%) were single, the remainder being married or divorced. Twenty (62.5\%) were university students or graduates. There were no significant differences in baseline demographics of the intervention and control groups.

\section{Post-treatment social conditioning outcomes: reading mind in eyes test}

Table 2 indicates that the total social cognition score as measured by the RMET was significantly greater following ISTDP than in the control group with a medium effect size. This significant increase was seen in both positive and neutral subjective state scores. By contrast, there was a significant reduction in the negative subjective state (score) in the posttest of the experimental group compared to the control group.

\section{Discussion}

Social cognition, the ability to identify and interpret other people's affect, mental states and actions, is an essential skill for maintaining interpersonal relationships (Pinkham et al., 2014). This capacity is often impaired in patients suffering from psychiatric disorders (Derntl et al., 2009; Wang et al., 2008; Weightman et al., 2014), which in turn affect patients' quality of life (Billeke et al., 2013; Cusi et al., 2012). Deficits in social cognition also lead to poor interpersonal relationships and an increased risk of relapse. As a result, any treatment that can improve social cognition should also improve patient outcome.

But even though social cognition is impaired in major depression, and even though we understand its neurological correlates, the ability to improve social cognition remains quite limited. Lahera et al.'s (2013) SCIT study was restricted to patients with bipolar and schizoaffective disorders. We therefore tested whether a psychodynamic psychotherapy that focused on improving affect awareness and internal 
Table 2. Outcome scores on social cognition.

\begin{tabular}{|c|c|c|c|c|c|c|c|c|c|c|}
\hline & \multicolumn{4}{|c|}{ Controls } & \multicolumn{4}{|c|}{ Experimental } & & \\
\hline & Mean & SD & Mean & SD & Mean & SD & Mean & SD & $F_{(1,28)}$ & Effect size \\
\hline \multicolumn{11}{|l|}{ Total } \\
\hline Total score & -14.33 & 45.67 & -30.64 & 42.62 & -22.31 & 29.67 & 21.97 & 22.10 & $48.31 * *$ & 0.63 \\
\hline Total time & 298.74 & 107.04 & 284.23 & 70.46 & 346.20 & 137.97 & 261.71 & 51.60 & 3.36 & 0.11 \\
\hline Indexes & & & & & & & & & $F_{(1,24)}$ & \\
\hline Negative subjective state (score) & 19.23 & 13.17 & 12.30 & 12.80 & 42.69 & 13.15 & -1.03 & 9.57 & $22.12 * *$ & 0.48 \\
\hline
\end{tabular}

$* p<0.05, * * p<0.01)$.

experience of feelings would lead to improved social cognition in interpersonal relationships as well.

In psychodynamic theory, we see three important relationships between affect tolerance and social cognition. First of all, following the theories of Wilfred Bion, emotional experience of reality is considered the substrate of cognitive thought. In other words, the preconscious, emotional, prereflective experience of reality is the precondition for thought. Second, patients who have a low tolerance for tolerating their feelings will become overwhelmed with anxiety. This anxiety, when channeled into the parasympathetic branch of the autonomic nervous system, impairs thinking processes in patients. Neurohormones start to impair the functioning of the prefrontal cortex, leading to a drop in metacognitive selfreflective function, and neurohormones also begin to shut down the hippocampus, leading to the problems in the storage of short-term memory into long-term memory. Third, patients with a low level of affect tolerance often project, their feelings are onto other people. Unable to tolerate their anger, they imagine others are angry with them.

In a previous article, we reported on how patients in this sample showed a sustained reduction in depression severity after receiving ISTDP along with improvements in executive functioning secondary to depression when compared with a wait-list control group (Ajilchi et al., 2016). In the current study, we assessed social cognition, which is abnormal emotional processing that is a core symptom of depression. The primary objective of the present article was therefore to examine whether the treatment of depression using psychodynamic therapy, specifically ISTDP, might alter social cognition. The experimental group showed a significant reduction in the negative subjective score compared to the control group. The experimental group also showed greater improvement in response to positive neutral and negative states. Consistent with our original hypotheses, our results indicate that ISTDP treatment caused modest improvements on overall scores of social cognition. Thus, depressed patients receiving ISTDP showed a significant improvement in social cognition post-treatment compared to a wait-list control group. To our knowledge, this is the first study to demonstrate the impact of psychotherapy on social cognition. This is important because improved outcome resulted from an intervention that is relatively brief and cost-effective.

Strengths of the study include the use of a randomized control design and standardized evaluation of social cognition before and after treatment within a naturalistic setting. As a result, these findings should have greater generalizability to routine practice. However, there are several limitations. These include the small sample size and lack of follow-up data on whether changes in social cognition were maintained. We were unable to conduct an ITT analysis because of missing data. It is also possible that the improvements in the ISTDP group could be due to the non-specific effects of increased health service contacts when compared to the wait-list controls, rather than the specific results of ISTDP.

In conclusion, these preliminary results need to be confirmed in a larger study with both the active and control groups unaware of allocation status and having similar clinic visits, even if the experimental group is receiving ISTDP and the control group receiving treatment as usual.

\section{Declaration of interest}

No potential conflict of interest was reported by the authors.

\section{References}

Abbass A, Town JM, Driessen E. (2012). Intensive short-term dynamic psychotherapy: A systematic review and meta-analysis of outcome research. Harv Rev Psychiatry, 20, 97-108.

Abbass A. (2015). Reaching through resistance. Kansas City, MO: Seven Leaves Press.

Ajilchi B, Nejati V, Town JM, et al. (2016). Effects of intensive short-term dynamic psychotherapy on depressive symptoms and executive functioning in major depression. J Nervous \& Mental Dis, 204, 500-5.

Baron-Cohen S, Wheelwright S, Hill J, et al. (2001a). The "reading the mind in the eyes" test revised version: A study with normal adults, and adults with Asperger syndrome or high-functioning autism. J Child Psychol Psychiatry, 42, 241-51.

Baron-Cohen S, Wheelwright S, Skinner R, et al. (2001b). The autism-spectrum quotient (AQ): Evidence from Asperger syndrome/high functioning autism, males and females, scientists and mathematicians. J Autism and Dev Disord, 31, 5-17.

Beevers C, Wells T, Ellis A, Fischer K. (2009). Identification of emotionally ambiguous interpersonal stimuli among dysphoric and non-dysphoric individuals. Cogn Ther Res, 33, 283-90.

Billeke P, Boardman S, Doraiswamy PM. (2013). Social cognition in major depressive disorder: A new paradigm? J Translational Neurosci, 4, 437-47.

Coughlin P. (2017). Maximizing effectiveness in dynamic psychotherapy. New York, NY: Routledge.

Cusi AM, Nazarov A, Holshausen K. (2012). Systematic review of the neural basis of social cognition in patients with mood disorders. J Psychiatry Neurosci, 37, 154-69.

Davanloo H. (2000). Intensive short-term dynamic psychotherapy: Selected papers of Habib Davanloo. Chichester: Wiley.

Davanloo H. (2005). Intensive short-term dynamic psychotherapy. In: Sadock BJ, Sadock VA, eds. Kaplan and Sadock's 
comprehensive textbook of psychiatry. Philadelphia: Lippincott Williams \& Wilkins, 2628-52.

Derntl B, Seidel EM, Kryspin-Exner I, et al. (2009). Facial emotion recognition in patients with bipolar I and bipolar II disorder. Br J Clin Psychol, 48, 363-75.

Gray J, Venn H, Montagne B, et al. (2006). Bipolar patients show moodcongruent biases in sensitivity to facial expressions of emotion when exhibiting depressed symptoms, but not when exhibiting manic symptoms. Cogn Neuropsychiatry, 11, 505-20.

Kaviani H, Mousavi A, Mohit A. (2001). Psychological interviews and tests. Tehran: Sana Publication. [Persian].

Kerr N, Dunbar RI, Bentall RP. (2003). Theory of mind deficits in bipolar affective disorder. J Affect Disord, 73, 253-9.

Lahera G, Benito A, Montes JM, et al. (2013). Social cognition and interaction training (SCIT) for outpatients with bipolar disorders. J Affect Disord, 146, 132-6.

Lee L, Harkness KL, Sabbagh MA, Jacobson JA. (2005). Mental state decoding abilities in clinical depression. J Affect Disord, 86, 247-58.

Leech NL, Barrett KC, Morgan GA. (2005). SPSS for intermediate statistics: Use and interpretation. Mahwah, NJ: Lawrence Erlbaum Associates.

Nejati V, Maleki G, Zabihzadeh A. (2012b). Mind reading and mindfulness deficit in patient with major depression disorder. J Social and Behavioral Sci, 32, 43.
Nejati V, Zabihzadeh A, Maleki GH, Mohseni M. (2013). Social cognition in patients with major depressive disorder: Evidence from the reading mind in the eyes test. J Applied Psychol, 4, 57-70.

Nejati V, Zabihzadeh A, Nikfarjam MR, et al. (2012a). Correlation between mindfulness and mind reading in the eye. Zahedan J. Res in Medical Sci, 14, 37-42. [In Persian].

Pelphrey K, Adolphs R, Morris JP. (2004). Neuroanatomical substrates of social cognition dysfunction in autism. Ment Retard Dev Disabil Res Rev, 10, 259-71.

Pinkham AE, Penn DL, Green MF, et al. (2014). The social cognition psychometric evaluation study: Results of the expert survey and RAND panel. Schizophr Bull, 40, 813-23.

Town JM, Driessen E. (2013). Emerging evidence for intensive shortterm dynamic psychotherapy with personality disorders and somatic disorders. Psychiatric Ann, 43, 502-7.

Wang YG, Wang YQ, Chen SL, et al. (2008). Theory of mind disability in major depression with or without psychotic symptoms: A componential view. J Psychiatry Res, 161, 153-61.

Weightman MJ, Air TM, Baune BT. (2014). A review of the role of social cognition in major depressive disorder. Front Psychiatry, 5, 179.

Weiner KS, Grill-Spector K. (2012). The improbable simplicity of the fusiform face area. Trends Cogn Sci, 16, 251-4. 


\section{Effects of intensive short-term dynamic psychotherapy on social cognition in major depression}

\section{Bita Ajilchi, Steve Kisely, Vahid Nejati \& Jon Frederickson}

To cite this article: Bita Ajilchi, Steve Kisely, Vahid Nejati \& Jon Frederickson (2018): Effects of intensive short-term dynamic psychotherapy on social cognition in major depression, Journal of Mental Health, DOI: 10.1080/09638237.2018.1466035

To link to this article: https://doi.org/10.1080/09638237.2018.1466035

曲 Published online: 23 May 2018.

Submit your article to this journal $\sqsubset$

Q View related articles $\square$

View Crossmark data $\subset$ 